\title{
La estructura factorial de la Escala hospitalaria de Ansiedad y Depresión en pacientes adolescentes con enfermedad crónica
} Factor structure of the Hospital Anxiety and Depression Scale in adolescent patients with chronic disease

a. Departamento de Personalidad, Evaluación y Tratamientos Psicológicos, Facultad de Psicología.

b. Facultad de Psicología.

c. Departamento de Psicología Social, Facultad de Psicología.

Universidad de

Valencia,

España.

Correspondencia:

Dra. Marián Pérez-

Marín:

marian.perez@uv.es.

Financiamiento:

El estudio ha sido realizado gracias a las becas de investigación recibidas por la Asociación Española de Psicología Clínica, Psicopatología y la Federación Española de Asociaciones de Terapia Familiar, el Programa VLCBIOMED (Instituto de Investigación en Fe, Hospital Universitario y Politécnico La Fe y Universitat de València), una Beca de la Generalitat Valenciana y el Fondo Social Europeo para la formación de personal investigador y docente, y una Beca de Iniciación a la Investigación de la Universitat de València.

Conflicto de intereses: Ninguno que declarar.

Recibido: $12-3-2018$

Aceptado: 7-2-2019

\author{
Lic. Selene Valero-Moreno ${ }^{a}$, Lic. Laura Lacomba-Trejo ${ }^{a}$, Lic. Sara Casaña-Granell ${ }^{b}$, \\ Dr. Vicente J. Prado-Gascóc, Dra. Inmaculada Montoya-Castilla ${ }^{a}$ y \\ Dra. Marián Pérez-Marín ${ }^{a}$
}

\section{RESUMEN}

Introducción. La Escala hospitalaria de Ansiedad y Depresión (Hospital Anxietyand Depression Scale, $H A D S$ ) es un instrumento de pesquisa, utilizado para evaluar sintomatología emocional en diferentes poblacionesy patologías médicas. Este estudio analiza las propiedades psicométricas y la estructura factorial de la $H A D S$ en adolescentes con enfermedad crónica y las diferencias en función de la patología médica.

Método.LaHADSfueadministradaaunamuestra de 302 adolescentes con enfermedad crónica. Se realizaron análisis factoriales exploratorios con una submuestra de 100 adolescentes y análisis factoriales confirmatorios con el resto de los participantes (202) para analizar la validez y fiabilidad de la HADS (14 ítems); además se calculó el análisis de la varianza de un factor para analizar las diferencias entre patologías. Resultados. Pacientes de 12 a 16 años de edad, con una distribución similar por género; el $43 \%$ padecía enfermedades respiratorias, y el $47 \%$, endocrinológicas. La estructura original de dos factores (ansiedad y depresión) fue la que mostró buenos índices de ajuste e incorporó un factor de $2^{\circ}$ orden (malestar emocional). Tres de los ítems originales de la escala fueron eliminados; se obtuvo, finalmente, una nueva versión de 11 ítems. Esta mostró adecuadas propiedades psicométricas y se observaron diferencias entre pacientes con diabetes mellitus 1 y con patología respiratoria, pero no en talla baja, en el factor de depresión.

Conclusiones. Esta escala presenta una adecuada fiabilidad y validez en pacientes con enfermedad crónica pediátrica y resulta útil para la detección temprana de clínica ansioso-depresiva en el ámbito sanitario.

Palabras clave:estructura factorial, cuestionario de salud del paciente en escala de ansiedad y depresión, diabetes mellitus tipo 1, enfermedades respiratorias crónicas, estatura baja.

http:/ / dx.doi.org/10.5546/ aap.2019.252

Texto completo en inglés:

http:/ / dx.doi.org/10.5546/ aap.2019.eng.252
Cómo citar: Valero-Moreno S, Lacomba-Trejo L, Casaña-Granell S, Prado-Gascó VJ, et al. La estructura factorial de la Escala hospitalaria de Ansiedad y Depresión en pacientes adolescentes con enfermedad crónica. Arch Argent Pediatr 2019;117(4):252-258

\section{INTRODUCCIÓN}

La presencia de una enfermedad crónica (EC) en la adolescencia supone una perturbación importante. ${ }^{1} \mathrm{La}$ literatura señala que los problemas de salud mental en la adolescencia pueden interferir negativamente en el tratamiento y en el curso de la enfermedad, lo que provoca complicaciones médicas en las EC. 2,3

Los adolescentes con problemas crónicos de salud física suelen referir síntomas ansioso-depresivos, ${ }^{4}$ que se asocian con mayor sintomatología conductual e hiperactividad ${ }^{5}$ y peor bienestar físico y psicológico. ${ }^{6}$

Dentro de la investigación en el contexto hospitalario, existe interés en identificar la clínica emocional para poder prevenir las complicaciones médicas que puedan surgir de ella. ${ }^{7}$ La Escala Hospitalaria de Ansiedad y Depresión (Hospital Anxiety and Depression Scale, $H A D S)^{8}$ es el instrumento más referido por la literatura científica y ampliamente utilizado para evaluar la sintomatología ansiosodepresiva en personas con EC.912 Está compuesta por 14 ítems, divididos en dos subescalas: ansiedad (HADS- $A$ ) y depresión (HADS-D), ${ }^{8}$ y obtiene un indicador de malestar emocional a partir del sumatorio de 
las puntuaciones de las subescalas. ${ }^{8}$ Entre las principales aportaciones de la HADS en cuanto a otras medidas de clínica ansioso-depresiva (Beck Depression Inventory -BDI- o Beck Anxiety Inventory-BAI-), ${ }^{13}$ está su enfoque centrado en los aspectos cognitivos frente a los somáticos, muy importante en los pacientes que presentan EC, cuyos síntomas podrían confundirse con la clínica ansioso-depresiva que acompaña la enfermedad propiamente dicha. ${ }^{8}$

La HADS se ha adaptado a múltiples idiomas ${ }^{11,12,14-16} \mathrm{y}$ aplicado a pacientes con distintas enfermedades físicas. ${ }^{9,11,16-21}$ Aunque, inicialmente, se desarrolló para personas de entre 16 y 65 años, ${ }^{9}$ se ha ampliado el rango de edad de su uso y se ha utilizado desde los 10 años hasta los 85. ${ }^{14,17,21-26}$ Esto se debe a la información que aporta, a su fácil comprensión y a la corta duración de su aplicación. ${ }^{27}$

Son numerosas las publicaciones que encuentran adecuadas propiedades psicométricas, ${ }^{21-23,28-30}$ que destacan índices de consistencia interna apropiados. ${ }^{26,30} \mathrm{Su}$ aplicación en niños y adolescentes, en poblaciones diferentes a la española, ha arrojado buenas propiedades psicométricas para la escala general de malestar emocional y para la $H A D S-A$, aunque menos potentes psicométricamente para la HADS-D. ${ }^{25,30}$ Asimismo, los estudios en esta población han mostrado como la HADS es un buen predictor para la clínica ansioso-depresiva. ${ }^{31}$

Respecto a su estructura factorial, existen diferencias en función de los métodos utilizados. ${ }^{24}$ No obstante, la mayoría destaca que está compuesta de dos factores tanto en los adultos ${ }^{10,21-23,29}$ como en los adolescentes. ${ }^{30-32}$ Aunque es una escala muy utilizada internacionalmente en clínica e investigación, su uso se ha limitado a los adultos. ${ }^{11,12,16,18}$ Asimismo, existen estudios realizados en adolescentes, pero en otros países. ${ }^{25,31}$ En la actualidad, en España, no existe literatura que estudie las propiedades psicométricas de esta escala en la población adolescente.

\section{Objetivos e hipótesis}

Los objetivos fueron analizar la estructura factorial de esta escala en una muestra de adolescentes con EC; comprobar las propiedades psicométricas y la dimensionalidad de la escala y evaluar las diferencias en la clínica ansiosodepresiva según el diagnóstico médico. En función de los objetivos, las hipótesis fueron $\mathrm{H} 1$ : la estructura de la escala sería bifactorial; y H2: los pacientes con patología respiratoria crónica (PRC) presentarían mayor clínica ansioso-depresiva respecto a sus iguales con diabetes y talla baja.

\section{MÉTODO \\ Participantes}

La muestra estuvo formada por un total de 378 participantes. De todos ellos, 76 fueron excluidos, al revisar el historial clínico de los pacientes entrevistados o al preguntar directamente al personal sanitario, porque no cumplían con los siguientes criterios de inclusión: pacientes adolescentes (12-16 años) diagnosticados, al menos, hacía 6 meses, que no presentaban un trastorno psicológico previo o trastorno por déficit de atención e hiperactividad (TDAH) o deterioro cognitivo o discapacidad intelectual.

\section{Instrumentos}

$H A D S:{ }^{8}$ Cuestionario de 14 ítems, con un formato de respuesta tipo Likert de 0 a 3, en el que 0 era mínima puntuación y 3, máxima puntuación; servía para detectar la sintomatología ansiosa (ítems impares) y depresiva (ítems pares) de tipo cognitivo en la última semana. El sumatorio de las dos escalas podía generar la puntuación global de malestar emocional. En general, mayores puntuaciones indicaban una mayor afectación emocional (mayores niveles de ansiedad y de depresión).

\section{Diseño}

Diseño transversal, aprobado por el Comité de Ética de la Universidad de Valencia y por los comités de los diferentes hospitales participantes. Los datos fueron recolectados entre 2015 y 2017 después de que los tutores firmaran el consentimiento informado. Los participantes pertenecían al Servicio de Endocrinología o Neumología Pediátrica correspondiente a hospitales de la Comunidad Valenciana (Hospital General Universitario, Hospital La Fe y Hospital Clínico Universitario). El pase de evaluación fue realizado por un psicólogo entrenado de forma estandarizada, y se aplicó el mismo protocolo en las tres patologías.

\section{Análisis de datos}

El análisis estadístico del instrumento se realizó utilizando SPSS 23.0, así como el software de modelos de ecuaciones estructurales (EQS, versión 6.3) y el de análisis factorial exploratorio (AFE; FACTOR 10.8.01). ${ }^{33}$ El AFE se llevó a cabo de acuerdo con el proceso recomendado por 
Lloret-Segura, ${ }^{34}$ utilizando el método de mínimos cuadrados no ponderados (ULS), la aplicación del método de análisis paralelo y la rotación directa de Oblimin, y fijando el número de factores a dos, tal como sugería la estructura factorial original del cuestionario. ${ }^{8}$

Las propiedades de los ítems fueron analizadas utilizando los coeficientes de correlación ítemtotal y variaciones en los coeficientes alfa de Cronbach si se eliminaban ítems. Las propiedades psicométricas fueron probadas por el AFE, el análisis factorial confirmatorio (AFC), la fiabilidad alfa y compuesta (CR) de Cronbach. Para el AFC, se utilizó la estimación de corrección robusta Satorra-Bentler $\left(S-B \chi^{2}\right)$ de máxima verosimilitud (MVR) para corregir la ausencia de normalidad multivariada. La idoneidad de los AFC se probó utilizando la significación del chi cuadrado y $S-B \chi^{2} \cdot{ }^{35}$

Los índices de bondad de ajuste de los modelos propuestos se probaron con el de ajuste no normalizado (Non Normed Fit Index; NNFI, por sus siglas en inglés), el de ajuste comparativo (Comparative Fit Index; CFI, por sus siglas en inglés) y la fijación de ajuste incremental (Incremental Fit Index; IFI, por sus siglas en inglés). Para estos indicadores, los valores superiores a 0,90 se consideraron adecuados. ${ }^{36}$ Además, se utilizó el error de aproximación medio de la raíz cuadrática (Root Mean Square Error of Approximation; RMSEA, por sus siglas en inglés) y se requirió que estas puntuaciones fueran inferiores a 0,08 para ser consideradas con un buen ajuste. ${ }^{37}$ Para llevar a cabo el AFE y el AFC, la muestra total $(n=302)$ se dividió en dos: el grupo A $(n=100)$ se utilizó para el AFE, y el grupo B $(n=202)$, para el AFC. Los grupos se seleccionaron aleatoriamente, teniendo en cuenta la proporcionalidad respecto a la patología médica, la edad y el sexo de los participantes. Se analizaron las diferencias de medias de las puntuaciones de la escala en función del diagnóstico (factor análisis de la varianza -analysis of the variance; ANOVA, por sus siglas en inglés-). Para estos análisis, se utilizó SPSS 23.0.

\section{RESULTADOS}

Los participantes fueron adolescentes con EC de 12-16 años (media $-\mathrm{M}-=13,52$; desviación típica -DT- $=1,21)$; el 58,3\% eran varones. Se obtuvo una muestra de 302 participantes: el $43 \%$ $(\mathrm{n}=130)$, con diagnóstico de patología respiratoria crónica (PRC), que fueron, mayoritariamente, asma bronquial $(\mathrm{n}=76)$ y bronquiolitis obliterante $(\mathrm{n}=14)$, $\mathrm{y}$, en menor medida, fibrosis quística $(n=7)$, discinesia ciliar primaria $(n=4)$ y otras enfermedades respiratorias, como malacias o bronquiectasias $(\mathrm{n}=29)$. El 25,2 \% $(\mathrm{n}=76)$ fueron pacientes con diabetes mellitus tipo 1 (DM1) y el 31,8\% (n=96), con talla baja como afección principal no secundaria a otra patología y de origen genético. Se seleccionaron estas patologías porque eran las principales EC en la etapa infantojuvenil, por lo que su aplicabilidad podía ser mayor.

El instrumento constaba de 14 ítems distribuidos en dos factores (ansiedad y depresión). Tras el análisis de los elementos, se suprimieron los ítems 7 y 14 porque así aumentaba el alfa en la dimensión global (malestar emocional). En general, todas las dimensiones tenían coeficientes aceptables, excepto en depresión, que era inferior a 0,70. Para verificar la distribución normal de la puntuación del ítem, se observaron los valores de asimetría y Curtosis, como se muestra en la Tabla 1. A menudo, eran mayores de 2 o -2, lo que no proporcionaba seguridad de la normalidad de la distribución de los datos.

Antes de llevar a cabo el AFE y el AFC, la adecuación de los datos fue determinada por la prueba de esfericidad de Kaiser-Meyer-Olkin $(\mathrm{KMO})$ y Bartlett. $\mathrm{KMO}(\mathrm{KMO}=0,73)$ y la prueba de esfericidad de Bartlett $\left(\chi^{2}=393,2 ; \mathrm{gl}=91\right.$; $p \leq, 001)$ presentaron valores adecuados, por lo que el AFE y el AFC se realizaron.

El AFE se calculó para el grupo A $(n=100)$ utilizando FACTOR con los 14 ítems. Al realizar el análisis paralelo, los datos, sin fijar el número de factores con autovalores igual a 1 , no mostraron acuerdo respecto al número de factores. Así, según el percentil 95, los ítems se agrupaban en una sola dimensión y, fijándose en la media, en dos dimensiones (ansiedad y depresión). Tras aplicar el AFE fijado a dos factores tal y como sugería la literatura de la escala original, se eliminaron aquellos ítems cuya saturación fue inferior a 0,40 o superior a este valor en más de un factor: 3 ítems $(4,7,14)$. Esta solución factorial presentaba buenos índices de ajuste $(R M S E A=0,05 ; C F I=0,98)$. La varianza explicada por estas dos dimensiones fue del $61,43 \%$. El análisis de correlaciones entre los elementos de la escala se indica en la Tabla 2.

Después de tener en cuenta los resultados del AFE, el AFC se llevó a cabo con el grupo B $(n=202)$. Los indicadores de bondad de ajuste para la solución de dos factores en la versión de 14 ítems fueron inapropiados, por lo que 
se eliminaron aquellos ítems que presentaban problemas en la carga factorial, $\leq 0,30$ en las puntuaciones, lo que incrementó los indicadores de bondad de ajuste del modelo cuando se eliminaron 3 ítems (ítems 4, 7, 14) (Tabla 3). Se repitió el mismo procedimiento para la solución de un único factor (malestar emocional); se eliminó el mismo número de ítems y se obtuvo que el modelo mejoraba. Los resultados indicaban que la solución bifactorial (ansiedad y depresión) y el modelo de un único factor (malestar emocional) mostraban buenos índices de ajuste del modelo (RMSEA $\leq 0,08 ;$ CFI y
IFI $\geq 0,90$ ), aunque el bifactorial mostraba índices ligeramente mejores. Por ello, ante las dudas del número de factores de la escala, se calculó un factor de $2 .^{\circ}$ orden (malestar emocional), y se lograron adecuados índices de ajuste de este modelo (Tabla 3). Finalmente, se obtuvo una escala reducida de 11 ítems (Tabla 4).

Los coeficientes de fiabilidad del alfa y el FC de Cronbach para cada dimensión variaron entre 0,78 y 0,84 , lo que se encontraba dentro de los valores mínimos $(\geq 0,70)$ indicados en la literatura, excepto para la dimensión de depresión, que mostraba niveles más bajos. La

TABLA 1. Análisis de ítems y fiabilidad de la Escala de Ansiedad y Depresión Hospitalaria en una muestra de adolescentes con enfermedad crónica

\begin{tabular}{|c|c|c|c|c|c|c|}
\hline & $\mathbf{M}$ & DT & rjx & $\alpha-\mathbf{x}$ & A & K \\
\hline \multicolumn{7}{|c|}{ Ansiedad: $\alpha=0,77 ; \alpha(\sin$ ítem HADS7) $=0,78 ; \mathrm{FC}=0,79 ; \mathrm{CI}=(0,74-0,82)$} \\
\hline HADS1 & 0,99 & 0,77 & 0,60 & 0,72 & 0,81 & 0,80 \\
\hline HADS3 & 0,57 & 0,88 & 0,55 & 0,73 & 1,32 & 0,53 \\
\hline HADS5 & 0,93 & 0,75 & 0,52 & 0,74 & 0,78 & 0,84 \\
\hline HADS7 & 1,14 & 0,85 & 0,29 & 0,78 & 0,25 & $-0,69$ \\
\hline HADS9 & 0,71 & 0,74 & 0,44 & 0,75 & 0,93 & 0,66 \\
\hline HADS11 & 1,03 & 0,84 & 0,50 & 0,74 & 0,55 & $-0,22$ \\
\hline HADS13 & 0,59 & 0,67 & 0,57 & 0,73 & 0,97 & 0,88 \\
\hline \multicolumn{7}{|c|}{ Depresión: $\alpha=0,61 ; \alpha(\sin$ ítems HADS4, HADS14) $=0,62 ; \mathrm{FC}=0,62 ; \mathrm{CI}=(0,55-0,68)$} \\
\hline HADS2 & 0,32 & 0,56 & 0,38 & 0,55 & 1,71 & 2,53 \\
\hline HADS4 & 0,19 & 0,52 & 0,26 & 0,59 & 3,25 & 11,89 \\
\hline HADS6 & 0,29 & 0,52 & 0,39 & 0,55 & 1,77 & 3,09 \\
\hline HADS8 & 0,49 & 0,67 & 0,33 & 0,57 & 1,38 & 1,91 \\
\hline HADS10 & 0,27 & 0,59 & 0,62 & 0,57 & 2,46 & 6,41 \\
\hline HADS12 & 0,27 & 0,55 & 0,45 & 0,52 & 2,17 & 4,89 \\
\hline HADS14 & 0,36 & 0,69 & 0,16 & 0,63 & 1,99 & 3,55 \\
\hline \multicolumn{7}{|c|}{ Malestar emocinal: $\alpha=0,80 ; \alpha(\sin$ ítems $4,7,14)=0,81 ; \mathrm{FC}=0,84 ; \mathrm{CI}=(0,78-0,84)$} \\
\hline
\end{tabular}

HADS: Escala de Ansiedad y Depresión Hospitalaria; M: media; DT: desviación típica; rjx: correlación ítem-total; $\alpha$-x: alfa de Cronbach si se elimina el ítem; A: asimetría; K: Curtosis; FC: fiabilidad compuesta;

CI: intervalo de confianza para alfa de Cronbach.

TABla 2. Autocorrelaciones de los componentes de la escala

\begin{tabular}{|c|c|c|c|c|c|c|c|c|c|c|c|c|c|c|}
\hline Ítems & 1 & 2 & 3 & 4 & 5 & 6 & 7 & 8 & 9 & 10 & 11 & 12 & 13 & 14 \\
\hline 1 & 1 & & & & & & & & & & & & & \\
\hline 2 & ,07 & 1 & & & & & & & & & & & & \\
\hline 3 & ,54 & ,37 & 1 & & & & & & & & & & & \\
\hline 4 & ,20 & 41 & ,29 & 1 & & & & & & & & & & \\
\hline 5 &, 54 & 31 &, 57 &, 30 & 1 & & & & & & & & & \\
\hline 6 & 48 & ,37 &, 50 &, 51 & ,37 & 1 & & & & & & & & \\
\hline 7 & ,24 & ,05 & 01 & ,01 & ,33 & ,06 & 1 & & & & & & & \\
\hline 8 & ,30 & 33 &, 53 & ,18 & ,26 & 12 & ,09 & 1 & & & & & & \\
\hline 9 & ,42 & ,23 & 40 & ,31 & ,31 & ,33 & ,07 & 22 & 1 & & & & & \\
\hline 10 & ,03 & ,33 & ,33 & ,03 & 12 & ,26 &,- 06 & 34 & 12 & 1 & & & & \\
\hline 11 & ,35 &,- 07 & 38 & 12 & 39 & 35 & ,38 & 24 & 20 & 26 & 1 & & & \\
\hline 12 & ,10 & ,53 & 43 & ,39 & ,15 & ,57 & -,13 & ,19 & 45 & , 58 &,- 16 & 1 & & \\
\hline 13 &, 58 & 42 & 70 & 44 &, 53 & 46 & ,11 & 63 & ,39 & ,27 & 33 & ,33 & 1 & \\
\hline 14 & 01 & ,39 & 17 & 11 & .01 & 10 &,- 05 & ,19 & 20 & ,44 & ,13 & ,02 & ,30 & 1 \\
\hline
\end{tabular}


correlación entre las dos dimensiones fue de 0,60. Por último, después de la eliminación de ítems, se establecieron los nuevos puntos de corte para diferenciar entre la ausencia, el caso probable y la presencia de un trastorno clínico, tal como indicaba la escala original ${ }^{8}$ (Tabla 5).

Para evaluar la existencia de diferencias entre los diagnósticos médicos, se realizó un ANOVA más una prueba post hoc Games-Howell, porque no se asumieron varianzas iguales. Se encontraron diferencias significativas para la puntuación total de la dimensión depresión entre pacientes con DM1 y respiratorios; los últimos mostraron puntuaciones más elevadas $(\mathrm{F}=3,27 ; p=0,04)$ y con un pequeño tamaño de efecto $\left(\eta^{2}=0,02\right)$.

\section{DISCUSIÓN}

Se ha explorado la estructura factorial de la HADS en una muestra de pacientes adolescentes (12-16 años) con EC, en su versión original. Al analizar la estructura factorial (objetivo 1), los resultados propuestos en el AFE planteaban controversia sobre la dimensionalidad de la escala (objetivo 2). Al eliminar los ítems que cargaban de manera errónea para conseguir cerrar un modelo que cumpliera con los índices de ajuste necesarios en el AFC, se optó por la solución bifactorial con un factor de $2^{\circ}$ orden, por lo que se aceptaría la H1 (la escala estaría conformada por una estructura bifactorial).

TABla 3. Resultados de los análisis factorial confirmatorio: indicadores de bondad de ajuste del análisis factorial confirmatorio para las soluciones bifactoriales y unifactorial

\begin{tabular}{|c|c|c|c|c|c|c|c|}
\hline Modelo & S-B- $\chi^{2}$ & ge & $\mathbf{p}$ & S-B $\chi^{2} /$ ge & CFI & IFI & RMSEA \\
\hline HADS 14 ítems (2 factores) & 166,74 & 76 & $<, 000$ & 2,19 & 0,78 & 0,78 & 0,08 \\
\hline HADS 14 ítems (1 factor) & 173,10 & 77 & $<, 000$ & 2,24 & 0,77 & 0,78 & 0,07 \\
\hline HADS 12 ítems (2 factores sin ítems 14, 7) & 90,68 & 53 & $<, 001$ & 1,71 & 0,89 & 0,89 & 0,06 \\
\hline HADS 12 ítems ( 1 factor sin ítems 14,4 ) & 132,39 & 54 & $<, 000$ & 2,45 & 0,80 & 0,81 & 0,08 \\
\hline HADS 11 ítems (2 factores sin ítems $14,7,4$ ) & 70,12 & 43 & $<, 001$ & 1,63 & 0,92 & 0,92 & 0,06 \\
\hline HADS 11 ítems (1 factor sin ítems14, 4, 7) & 74,63 & 44 & $<, 003$ & 1,70 & 0,91 & 0,91 & 0,06 \\
\hline HADS 11 ítems ( 2 factores con factor de $2^{\circ}$ orden $)$ & 72,01 & 42 & $<, 002$ & 1,71 & 0,91 & 0,91 & 0,06 \\
\hline
\end{tabular}

HADS: Escala de Ansiedad y Depresión Hospitalaria; S-B- $\chi 2$ : Escala Satorra-Bentler chi-cuadrado; ge: grados de libertad; S-B $\chi^{2}$ / ge: ratio entre S-B $\chi 2$ \& gl; CFI: índice comparativo de ajuste; IFI: índice de ajuste de Bollet; RMSEA: error cuadrático de aproximación.

TABla 4. Versión reducida de la Escala de Ansiedad y Depresión Hospitalaria en la muestra de pacientes pediátricos del estudio al eliminar los items

\section{Escala reducida de Ansiedad y Depresión Hospitalaria (HADS)}

Me siento tenso y nervioso (ítem 1).

Sigo disfrutando con las mismas cosas de siempre (ítem 2).

Siento una especie de temor como si algo malo fuera a suceder (ítem 3).

Tengo la cabeza llena de preocupaciones (ítem 5).

Me siento alegre (ítem 6).

Me siento lento/a y torpe (ítem 8).

Experimento una desagradable sensación de "nervios y hormigueos" en el estómago (ítem 9).

He perdido el interés por mi aspecto personal (ítem 10).

Me siento inquieto/a como si no pudiera parar de moverme (ítem 11).

Espero las cosas con ilusión (ítem 12).

Experimento de repente sensaciones de gran angustia o temor (ítem 13).

TABla 5. Nuevos puntos de corte para la interpretación de las puntuaciones de la Escala de Ansiedad y Depresión Hospitalaria en adolescentes

\begin{tabular}{lcc}
\hline Factor ansiedad & Factor depresión & Malestar emocional \\
\hline 0-6: normal-ausencia de ansiedad & $0-5,4:$ normal-ausencia de depresión & $<15,5:$ ausencia de diagnóstico clínico \\
de malestar emocional & $\geq 15,5:$ problema clínico de malestar \\
7-9: caso probable de ansiedad & $5,5-7,5:$ caso probable de depresión & emocional \\
$>$ 9: problema clínico de ansiedad & $>7,5:$ problema clínico de depresión & \\
\hline
\end{tabular}


La mayoría de los estudios van en la línea de una solución bifactorial, 9,18,20,21,29 aunque, debido a las dudas acerca de la dimensionalidad de la escala, se plantea un modelo bifactorial con un factor de $2^{\circ}$ orden (malestar emocional), y se señala que, en muestras adolescentes (adolescencia temprana y tardía), este tipo de trastornos emocionales (ansiedad y depresión) ${ }^{2}$ pueden ser difíciles de diferenciar.

En definitiva, el número final de ítems, al realizar los análisis de validez y fiabilidad, fue de 11. Eliminando los ítems 4, 7 y 14 por sus cargas anómalas, ${ }^{23}$ se obtuvieron adecuados índices de fiabilidad, excepto en depresión, como ocurría en estudios anteriores..$^{24,30} \mathrm{El}$ ítem 14 fue el que mostraba mayor problemática respecto a las cargas factoriales. Como ya sucedía en algunas de las adaptaciones estudiadas, ${ }^{22}$ posiblemente, podría haber perdido su relevancia cultural y su capacidad para evaluar anhedonia por tener, también, poca capacidad discriminativa. Además de la eliminación de este ítem, se eliminaron dos más que ayudaron a mejorar la estructura y la consistencia interna de la HADS.

Respondiendo al objetivo 3, se observaron diferencias en los niveles de depresión entre dos grupos de enfermedades: PRC y DM1. Se aceptó parcialmente la $\mathrm{H} 2$, ya que los pacientes con diagnóstico de enfermedad respiratoria mostraban, por lo general, más problemas emocionales. $^{2}$

Nuestros resultados permiten ir en la línea del uso de esta escala en la población con patologías médicas crónicas, pues amplía su aplicación con adecuadas propiedades psicométricas en la población adolescente, sirve como instrumento de pesquisa y ofrece la oportunidad de planificar intervenciones dirigidas a esta población. Además, otro aspecto relevante es que ha permitido evaluar, a la vez, la clínica ansioso-depresiva de tres tipos diferentes de patologías no evaluadas con anterioridad, como la DM1, la talla baja y los PRC. No obstante, son necesarios más estudios que comprueben sus propiedades psicométricas en muestras de adolescentes con EC para aportar mayor evidencia acerca de su funcionamiento.

A pesar de las contribuciones señaladas anteriormente, los resultados solo pueden considerarse preliminares debido a las limitaciones del presente estudio. Sería recomendable aumentar la muestra de pacientes adolescentes; no obstante, en general, en este tipo de estudios hospitalarios, las muestras finales obtenidas suelen ser reducidas, debido a la dificultad de recoger una muestra de pacientes adolescentes con una EC. Otra de las limitaciones radica en la utilización únicamente de datos provenientes de cuestionarios; se recomendaría el uso de indicadores médicos (tipo de tratamiento médico, nivel de cortisol, número de hospitalizaciones) para contrastar los datos del cuestionario. Además de contar con otros informadores, como los cuidadores principales o el personal sanitario, para comprobar la correspondencia entre la percepción del paciente y su entorno más cercano. Todas estas limitaciones se tendrán en cuenta en futuras investigaciones.

La Organización Mundial de la Salud y la Asociación Americana de Psiquiatría sugieren la necesidad de enfocar la adaptación a la EC en la adolescencia no solo desde el punto de vista médico, sino de manera multidimensional, lo que refleja la necesidad de construir instrumentos válidos para este tipo de poblaciones. Por tanto, este estudio resulta importante porque ha conseguido comprobar la estructura factorial de un cuestionario ampliamente utilizado a nivel internacional, pero que, hasta el momento, no se había realizado en la población adolescente española. Así, se obtiene una herramienta diagnóstica útil y de fácil interpretación para la detección temprana de sintomatología emocional (ansiedad y depresión) en las poblaciones adolescentes con patologías crónicas dentro del ámbito sanitario, que permitirá guiar intervenciones futuras con el fin de aumentar el bienestar emocional de los pacientes.

\section{REFERENCIAS}

1. Gómez-Rico I, Pérez-Marín M, Montoya-Castilla I. Diabetes mellitus tipo 1: breve revisión de los principales factores psicológicos asociados. An Pediatr (Barc). 2015; 82(1):e143-6.

2. Dudeney J, Sharpe L, Jaffe A, Jones EB, et al. Anxiety in youth with asthma: A meta-analysis. Pediatr Pulmonol.2017; 52(9):1121-9.

3. Korczak DJ, Madigan S, Manassis K, Daneman D. The association of corticol stress response with early adversity and diabetes control in adolescents with diabetes. J Depress Anxiety. 2016; 5(1):1000217.

4. Delamater AM, Guzman A, Aparicio K. Mental health issues in children and adolescents with chronic illness. Int J Hum Rights Healthc. 2017; 10(3):163-73.

5. Gómez-Restrepo C, Ramírez S, Tamayo Martínez N, Rodriguez MN, et al. Prevalencia de posibles trastornos mentales en niños con condiciones crónicas. Resultado de la Encuesta Nacional de Salud Mental Colombia 2015. Rev Colomb Psiquiatr. 2016; 45(S1):135-40.

6. Verhoof EJ, Maurice-Stam H, Heymans HS, Evers AW, et al. Psychosocial well-being in young adults with chronic illness since childhood: the role of illness cognitions. Child Adolesc Psychiatry Ment Health. 2014; 8:12. 
7. Quesada-Conde A, Justicia-Díaz MD, Romero-López M, García-Berbén MT. La enfermedad crónica infantil. repercusiones emocionales en el paciente y en la familia. Rev Infad. 2014; 4(1):569-76.

8. Zigmond AS, Snaith RP. The Hospital Anxiety and Depression Scale. Acta Psychiat Scand.1983; 67(6):361-70.

9. Lin CY, Pakpour AH. Using Hospital Anxiety and Depression Scale (HADS) on patients with epilepsy: Confirmatory factor analysis and Rasch models. Seizure. 2017; 45:42-6.

10. Terol-Cantero MC, Cabrera-Perona V, Martín-Aragón M. Revisión de estudios de la Escala de Ansiedad y Depresión Hospitalaria (HADS) en muestras españolas. Anal Psicol. 2015; 31(2):494-503.

11. Yang Y, Ding R, HuD, Zhang F, et al. Realibility and validity of Chinese version of the HADS for screening depression and anxiety in psycho-cardiological outpatients. Compr Psychiatry. 2014; 55(1):215-20.

12. Risal A, Manandhar K, Linde M, Koju R, et al. Reliability and Validity of a Nepali-language Version of the Hospital Anxiety and Depression Scale (HADS). Kathmandu Univ Med J (KUMJ). 2015; 13(50):115-24.

13. Beck JS, Beck AT, Jolly JB, Steer RA. The Beck Youth Inventories. 2nd ed. San Antonio: Psychological Corporation; 2005.

14. Aherrera JAM, Abrahan LL, Racaza GZ, Train CQ, et al. Depression and anxiety in adults with congenital heart disease using the validated filipino version of the hospital anxiety and depression score (HADS-P). Phillippine J Intern Med. 2016; 54(1):1-8.

15. Miljanović M, Sindik J, Milunović V, Škoc VK, et al. Factor structure and cut-off scores of the hospital anxiety and depression scale (HADS) in a Croatian sample of adult patients suffering from advanced cancer. Psychiatr Danub. 2017; 29(4):451-8.

16. Macêdo EA, Appenzeller S, Costallat LTL. Depression in systemic lupus erythematosus: gender differences in the performance of the BeckDepression Inventory (BDI), Center for Epidemiologic Studies Depression Scale (CES-D), and Hospital Anxiety and Depression Scale (HADS). Lupus. 2018; 27(2):179-89.

17. Djukanovic I, Carlsson J, ÅrestedtK. Is the Hospital Anxiety and Depression Scale (HADS) a valid measure in a general population 65-80 years old? A psychometric evaluation study. Health Qual Life Outcomes. 2017;15(1):193.

18. Martínez-López P, Durá-Ferrandis E, Andreu-Vaillo Y, Galdón-Garrido MJ, et al. Structural validity and distress screening potential of the Hospital Anxiety and Depression Scale in cancer. Int J Clin Health Psychol. 2012; 12(3):435-47.

19. Kaur S, Zainal NZ, Low WY, Ramasamy R, et al. Factor structure of Hospital Anxiety and Depression Scale in Malaysian patients with coronary artery disease. Asia Pacific J Public Health. 2015; 27(4):450-60.

20. Cabrera V, Martín-Aragón M, Terol MC, Núñez R, et al. La escala de ansiedad y depresión hospitalaria (HAD) en fibromialgia: análisis de sensibilidad y especificidad. Ter Psicol. 2015; 33(3):181-93.

21. Stott J, Spector A, OrrelL M, Scior K, et al. Limited validity of the Hospital Anxiety and Depression Scale (HADS) in dementia: evidence from a confirmatory factor analysis. Int J Geriatr Psychiatry. 2017; 32(7):805-13.
22. Monsalve V, Soriano J, Ibañez E. Fiabilidad y estructura factorial de la escala de ansiedad y depresión hospitalaria (HADS) en una muestra de pacientes con dolor crónico. Bol Psicol. 2001; 72:7-21.

23. Spurgeon L, James G, Sackley C. The Hospital Anxiety and Depression Scale: a pilot study to examine its latent structure and the link between psychological state and symptom severity in transient ischemic attack patients. Psychol Health Med. 2016; 21(5):632-8.

24. Saez-Flores E, Tonarely NA, Barker DH, Quittner AL. Examining the stability of the hospital anxiety and depression scale factor structure in adolescents and young adults with cystic fibrosis: a confirmatory factor analysis. J Pediatr Psychol. 2018; 43(6):625-35.

25. Ibáñez E, Caro I. La escala Hospitalaria de ansiedad y depresión. Su utilidad práctica en Psicología de la Salud. Bol Psicol. 1992; 36:43-69.

26. Fong TC, Ho RT. Factor analyses of the Hospital Anxiety and Depression Scale: A Bayesian structural equation modeling approach. Qual Life Res. 2013; 22(10):2857-63.

27. Turk DC, Dworkin RH, Trudeau JJ, Benson C, et al. Validation of the Hospital Anxiety and Depression Scale in patients with acute low back pain. J Pain. 2015; 16(10):1012-21.

28. Hinz A, Brähler E. Normative values for the Hospital Anxiety and Depression Scale (HADS) in the general German population. J Psychosom Res. 2011; 71(2):74-8.

29. Meneghetti CC, Guidolin BL, Zimmermann PR, Sfoggia A. Screening for symptoms of anxiety and depression in patients admitted to a university hospital with acute coronary syndrome. Trends Psychiatry Psychother. 2017; 39(1):12-8.

30. Mihalca AM, PileckaW. The factorial structure and validity of the Hospital Anxiety and Depression Scale (HADS) in Polish adolescents. Psychiatr Pol. 2015; 49(5):1071-88.

31. Ander M, Grönqvist H, Cernvall M, Engvall G, et al. Development of health-related quality of life and symptoms of anxiety and depression among persons diagnosed with cancer during adolescence: a 10-year follow-up study. Psychooncology. 2016; 25(5):582-9.

32. Kwong KL, Lam D, Tsui S, Ngan M, et al. Anxiety and Depression in Adolescents With Epilepsy. J Child Neurol. 2016; 31(2):203-10.

33. Lorenzo-Seva U, Ferrando PJ. FACTOR: A computer program to fit the exploratory factor analysis model. Behav Res Methods. 2006; 38(1):88-91.

34. Lloret-Segura S, Ferreres-Traver A, Hernández-Baeza A, Tomás-Marco I. El análisis factorial exploratorio de los ítems: una guía práctica, revisada y actualizada. An Psicol. 2014; 30(3):1151-69.

35. Satorra A, Bentler PM. Corrections to test stadistics and standard errors in covariance structure analysis. En: Von Eye A, Clogg CC (eds.). Latents variable analysis: applications to developmental research. Thousand OAKS, CA:SAGE; 1994.

36. MacCallum RC, Austin JT. Applications of structural equation modeling in psychological research. Annu Rev Psychol. 2000; 51:201-26.

37. Browne MW, Cudeck R. Alternative ways of assessing model fit. En: Bollen KA, Long JS (eds.). Testing structural equation models. Newbury Park, CA: SAGE; 1993. 